\title{
Preschoolers' Technology-Assessed Physical Activity and Cognitive Function: A Cross-Sectional Study
}

\author{
Minghui Quan ${ }^{1}{ }^{(1)}$, Hanbin Zhang ${ }^{1}$, Jiayi Zhang ${ }^{2}$, Tang Zhou ${ }^{1}$, Jinming Zhang ${ }^{3}$, \\ Guanggao Zhao ${ }^{4}$, Hui Fang ${ }^{1}$, Shunli Sun ${ }^{1}$, Ru Wang ${ }^{1, *}$ and Peijie Chen ${ }^{1, *}$ \\ 1 School of Kinesiology, Shanghai University of Sport, Shanghai 200438, China; \\ quanminghui@163.com (M.Q.); zhb20092009@163.com (H.Z.); tzhou_1023@foxmail.com (T.Z.); \\ ffanghui@163.com (H.F.); suns1087@outlook.com (S.S.) \\ 2 Editorial Department of Medicine and Health, China Science Publishing and Media Ltd., \\ Shanghai 200032, China; jiayi_0827@126.com \\ 3 Department of Kinesiology, College of Sport Medicine and Rehabilitation, Taishan Medical University, \\ Taian 271016, China; jmzhang@tsmc.edu.cn \\ 4 Department of Physical Education, Nanchang University, Nanchang 330031, China; zhaogg2002@163.com \\ * Correspondence: wangru0612@163.com (R.W.); chenpeijie@sus.edu.cn (P.C.); \\ Tel.: +86-21-5125-3240 (R.W.); +86-21-5125-3003 (P.C.)
}

Received: 19 April 2018; Accepted: 7 May 2018; Published: 8 May 2018

\begin{abstract}
Early childhood is a critical period for development of cognitive function, but research on the association between physical activity and cognitive function in preschool children is limited and inconclusive. This study aimed to examine the association between technology-assessed physical activity and cognitive function in preschool children. A cross-sectional analysis of baseline data from the Physical Activity and Cognitive Development Study was conducted in Shanghai, China. Physical activity was measured with accelerometers for 7 consecutive days, and cognitive functions were assessed using the Chinese version of Wechsler Young Children Scale of Intelligence (C-WYCSI). Linear regression analyses were used to assess the association between physical activity and cognitive function. A total of 260 preschool children (boys, 144; girls, 116; mean age: $57.2 \pm 5.4$ months) were included in analyses for this study. After adjusting for confounding factors, we found that Verbal Intelligence Quotient, Performance Intelligence Quotient, and Full Intelligence Quotient were significantly correlated with light physical activity, not moderate to vigorous physical activity, in boys. Standardized coefficients were $0.211,0.218$, and 0.242 (all $p<0.05$ ) in three different models, respectively. However, the correlation between physical activity and cognitive functions were not significant in girls $(p>0.05)$. These findings suggest that cognitive function is apparently associated with light physical activity in boys. Further studies are required to clarify the sex-specific effect on physical activity and cognitive functions.
\end{abstract}

Keywords: motor activity; intelligence quotient; young children

\section{Introduction}

Cognitive function is the ability to process information acquired from individual surroundings by the brain and includes the ability to learn and remember information, organize, plan and problem-solve, focus, maintain and shift attention, and understand and use language [1]. The stage of childhood is a critical period to develop cognitive function, as cognitive function in childhood may be an important indicator for future physical health, mental health, wealth, and public safety [2]. Therefore, identifying related factors in the development of cognitive function in childhood has drawn researchers' attention due to its substantial benefits extending into adulthood. 
Physical activity (PA), a component of lifestyle, is considered to be a potentially important factor in altering our brain health and mental function [3]. In recent years, many studies have examined the effects of PA on cognitive function. There is an increasing body of evidence suggesting that in children and adolescents, PA, particularly moderate to vigorous physical activity (MVPA), is closely associated with cognitive functions [4,5]. Furthermore, a systematic review concluded that there is a positive association between PA and cognitive function in children [6]. Animal evidence suggests that aerobic exercise can enhance human brain structure, prevent age-related brain tissue loss, and improve cognitive performance [7], so increased PA can enhance brain function. Previous human studies also indicated that PA in schools may enhance academic attainment, and higher levels of physical fitness in children may be associated with improved neurocognitive processing [8]. Increased physical activity may therefore provide cognitive and educational benefits across childhood and adolescence. So, moderate and vigorous PA was recommended for children and adolescents, and the importance of establishing healthy and appropriate behaviors in children is crucial for long-term effects. However, different types, amounts, and frequencies of PA were adopted in these studies. Moreover, in preschool stage, a key period for personality development, to the best of our knowledge, studies assessing the association between PA and cognitive functions is limited. Furthermore, previous trials were individually underpowered or primarily of weak quality to address this issue, and the few observational studies addressing it have mixed results [9].

Therefore, in the current study, the aim is to examine the PA conditions in preschool children and the association between the technology-assessed PA and cognitive functions, while adjusting for confounding factors that associated with cognitive function on the basis of previous studies, such as children's cardiorespiratory fitness, daily behavior, and mother's education $[6,10,11]$.

\section{Methods}

\subsection{Participants}

This cross-sectional study is a baseline data analysis from The Physical Activity and Cognitive Function Study (Trial Registration: ChiCTR-OOC-15007439) [12]. A total of 346 (boys, 201; girls, 145) preschool children were recruited from seven urban kindergartens in Shanghai, China. All the parents/guardians of potential participants have been fully informed of the protocol and aims of the study by parents' meeting held in the kindergarten. Signed informed consent forms were obtained from parents/guardians of the participants before this study began. The protocol was also approved by The Ethics Advisory Committee of Shanghai University of Sport.

\subsection{Measures and Procedures}

Participants' cognitive functions were evaluated using a short form of the Chinese version of Wechsler Young Children Scale of Intelligence (C-WYCSI) [13], due to constraints in assessment time. The short form consisted of four subtests, taking approximately $30 \mathrm{~min}$ to complete, and was also widely adopted in previous studies investigating cognitive function [14,15]. Furthermore, the short form of C-WYCSI was validated and the association of its scores and estimated Full Intelligence Quotient (FIQ) was also confirmed in our pilot study for preschool children $(n=31, r=0.90, p<0.01)$. The short-form items consisted of two tests: the Verbal Intelligence Quotient (VIQ: Information and Vocabulary) test and the Performance Intelligence Quotient (PIQ: Picture Completion and Block Design) test. The Information subtest involved asking participants to answer questions about everyday knowledge; participants received a 1 or 0 score for each correct or incorrect answer (total, 0-23 scores). For the Vocabulary subtest, children were asked to identify the true answer from four pictures corresponding to the word instructed by the tester; children scored 1 or 0 for each correct or incorrect response (total, $0-44$ scores). The Picture Completion subtest required children to identify and point out the missing part of the picture; children received a 1 or 0 score for each correct or incorrect answer (total, 0-25 scores). Finally, the Block Design subtest included a design either from the tester or the 
test booklet; children scored from 0 to 4 depending on how quickly they completed each design (total, 0-29 scores). After assessment, raw scores were converted to standard scores based on the instruction manual. Standardized scores of VIQ and PIQ were equal to the sum of two Verbal and Performance subtest scores, respectively. FIQ was estimated using weighted scores of each subtest according to the instruction manual (normal mean $=100, \mathrm{SD}=15.0$ ). Children were divided into five groups based on their FIQ scores: significantly below normal, slightly below normal, normal, slightly above normal, and significantly above normal were defined as $<70,70-<90,90-110,>110-130$, and $>130$ scores, respectively.

Physical activity was measured over 7 consecutive days during waking hours with ActiGraph accelerometers $\left(\mathrm{GT}_{3} \mathrm{X}^{+}\right.$, ActiGraph, Pensacola, FL, USA) on the right hip. The activity was captured by a $1 \mathrm{~s}$ sampling interval and categorized as either light or moderate to vigorous PA (LPA, MVPA) based on cutoff counts developed by Pate and colleagues for preschool children [16]. LPA corresponded with 101-1679 counts per minute (CPMs) and MVPA was equal or greater than 1680 CPMs. Individual data were validated into analyses when participating in at least 3 days (including 1 weekend day) of monitored PA, with a minimum of $8 \mathrm{~h}$ each day [17].

Children characteristics included sex, ages (months), heights, body weights, mother's education, family structures, and household income. Body mass index (BMI) was calculated using the formula weight $/$ height $^{2}\left(\mathrm{~kg} / \mathrm{m}^{2}\right)$ and BMI status was classified as normal, overweight, and obese using the cut points developed by International Obesity Task Force (IOTF) [18]. Mother's education was considered as a critical influence on children's cognitive function [11], and the education level was divided into six groups: less than high school, high school, some college/associate's degree, bachelor degree, master's degree, and doctor degree. "Living with both parents" and "living only with mother or father, or other situation" was used to evaluate family support. Household income was divided into six groups according to median household incomes in China: None, $<4000 \mathrm{RMB} /$ month, 4000-8000 RMB/month, 8001-15,000 RMB/month, 15,001-30,000 RMB/month, and $>30,000 \mathrm{RMB} /$ month $(1 \mathrm{RMB} \approx 0.16$ US dollars).

Children's daily behavior was an important indicator of physical activity [19] and was associated with cognitive functions [10]. Therefore, it was included as a covariate in the regression models. In this study, the past 2 months of children's daily behavior were measured using four items from the Chinese Child Behavior Checklist for Preschool Children. The items, completed by their teacher, were as follows: (1) whether the child shows lack of concentration or non-persistent attention; (2) whether the child is introverted and unwilling to talk; (3) whether the child is over-fatigued; and (4) whether the child has slow actions or anenergia. Items were rated by a 3 -point scale $(0=$ not true, $1=$ sometimes true, or 2 = often true). Finally, children's behavior scores were divided into three groups based on the total scores: low (4-6 scores), median (7-9 scores), and high (10-12 scores).

Cardiorespiratory fitness was assessed by the multistage $20 \mathrm{~m}$ shuttle run test, which measured cardiorespiratory fitness by running back and forth for $20 \mathrm{~m}$ with a starting speed of $8.5 \mathrm{~km} / \mathrm{h}$ and increasing by $0.5 \mathrm{~km} / \mathrm{h}$ with each level thereafter $(1 \mathrm{~min})$. Maximal performance was determined when the participant failed to follow the pace for two consecutive attempts or stopped due to exhaustion. Results were expressed as laps; one lap corresponded to $20 \mathrm{~m}$. The multistage $20 \mathrm{~m}$ shuttle run test is widely used for assessing cardiorespiratory fitness in preschool children and has shown to have a high reliability [20]. Due to the young age of participants, each child had an adult running with them during the process to make sure the test was successfully completed.

\subsection{Statistical Analyses}

Analyses were performed using SPSS version 22.0 (SPSS Inc., Chicago, IL, USA). The normal distribution test was conducted using Kolmogorov-Smirnov test, and variables were described as mean \pm SD for the normally distributed variables or median (interquartile ratio, IQR) for nonnormally distributed variables. Independent $t$ tests, Mann-Whitney $U$ tests, or chi-square tests 
were used to assess sex differences for normally distributed, non-normally distributed, or categorical variables, respectively.

Linear regression analyses were used to explore the association between different intensities of PA and cognitive functions. Variables were transformed to normal distribution using the log or square root method before linear regression, if necessary. To understand total variance explained by different factors, cognitive function regressed in three models: Model 1 LPA and MVPA entered the model and was unadjusted for confounding factors; Model 2 was adjusted for sociodemographic and children's daily behavior (including age, BMI status, mother's education, family structure, household income, and child behavior scores); Model 3 was further adjusted for cardiorespiratory fitness. Because PA and physical fitness have been suggested to be strongly linked with sex, our linear regression analyses were stratified for sex. Furthermore, to test the robustness and avoid high correlation between LPA and MVPA causing confusion of our results, LPA, MVPA, and total time engaged in physical activity (TPA, equal to sum of LPA and MVPA) were separately entered into the regression model again following the three steps described above. A two-sided $p$ value $\leq 0.05$ was considered as statistically significant.

\section{Results}

A total of 325 of 346 participants completed the C-WYCSI test. Among them, 11 preschool children were excluded from analyses because of noncooperation during the test $(n=6)$ or intellectual disability ( $n=5$, FIQ: 54.2-73.8). Characteristics of 260 participants (144 boys; 116 girls) who have complete data of cognitive functions, PA, and confounding factors are shown in Table 1. On average, the age of participants was 57.2 months (55.4\% boys), the majority (79.2\%) of preschool children were considered as healthy weight according to IOTF, and the percentage of overweight/obesity, LPA, and MVPA in boys were significantly higher than girls. However, the correlation between physical activity and cognitive functions were not significant in girls $(p>0.05)$.

Table 1. Characteristics of the analyzed sample.

\begin{tabular}{|c|c|c|c|c|}
\hline & Total $(n=260)$ & Boys $(n=144)$ & Girls $(n=116)$ & $p$ for Sex \\
\hline Age (month) & $57.2 \pm 5.4$ & $57.6 \pm 5.4$ & $56.7 \pm 5.3$ & 0.200 \\
\hline BMI $\left(\mathrm{kg} / \mathrm{m}^{2}\right)$ & $16.2 \pm 1.9$ & $16.5 \pm 1.9$ & $15.9 \pm 1.8$ & 0.001 \\
\hline Normal & 206 & 106 & 100 & \\
\hline Overweight/Obesity & 54 & 38 & 16 & 0.013 \\
\hline Mother's education & & & & 0.236 \\
\hline Less than high school & 10 & 3 & 7 & \\
\hline High school & 44 & 28 & 16 & \\
\hline College/associate degree & 82 & 42 & 40 & \\
\hline Bachelor's degree & 94 & 57 & 37 & \\
\hline Master's degree & 19 & 8 & 11 & \\
\hline Doctor degree & 11 & 6 & 5 & \\
\hline Family structure & & & & 0.502 \\
\hline Living with both parents & 251 & 140 & 111 & \\
\hline Others & 9 & 4 & 5 & \\
\hline Household income (RMB/month) & & & & 0.866 \\
\hline$<4000$ & 5 & 3 & 2 & \\
\hline $4000-8000$ & 42 & 22 & 20 & \\
\hline $8001-15,000$ & 115 & 65 & 50 & \\
\hline $15,001-30,000$ & 80 & 46 & 34 & \\
\hline$>30,000$ & 18 & 8 & 10 & \\
\hline Child behavior scores (count) & & & & $<0.001$ \\
\hline Low (4-6 scores) & 165 & 77 & 88 & \\
\hline Median (7-9 scores) & 88 & 60 & 28 & \\
\hline High (10-12 scores) & 7 & 7 & 0 & \\
\hline Cardiorespiratory Fitness (lap) & $11.0(10-14)$ & $11(9.25-14.0)$ & $12(10.0-14.75)$ & 0.328 \\
\hline
\end{tabular}


Table 1. Cont.

\begin{tabular}{lcccc}
\hline & Total $(\boldsymbol{n}=\mathbf{2 6 0})$ & Boys $(\boldsymbol{n}=\mathbf{1 4 4})$ & Girls $(\boldsymbol{n}=\mathbf{1 1 6})$ & $\boldsymbol{p}$ for Sex \\
\hline Physical activity (min/day) & & & & \\
LPA & $98.4 \pm 17.1$ & $100.6 \pm 17.9$ & $95.6 \pm 15.7$ & $\mathbf{0 . 0 2 1}$ \\
MVPA & $71.8 \pm 17.3$ & $74.1 \pm 18.7$ & $69.0 \pm 15.0$ & $\mathbf{0 . 0 2 1}$ \\
\hline Cognitive function & & & \\
VIQ & $23(19.0-26.0)$ & $22(19.0-26.0)$ & $23(19.0-26.0)$ & 0.558 \\
FIQ & $25(22.0-27.0)$ & $25(21.0-27.0)$ & $25(23.0-27.75)$ & 0.255 \\
FIQ & $110.5 \pm 12.4$ & $110.0 \pm 13.0$ & $111.3 \pm 11.6$ & 0.370 \\
\hline
\end{tabular}

Note: BMI, body mass index; LPA, light physical activity; MVPA, moderate to vigorous physical activity; VIQ, Verbal Intelligence Quotient; PIQ, Performance Intelligence Quotient; FIQ, Full Intelligence Quotient. The mean \pm SD or median (interquartile ratio, IQR) was reported for normal or non-normal distribution variables.

The results showed that LPA, but not MVPA, is significantly correlated with VIQ, PIQ, and FIQ, even when adjusting for several potential confounding factors in the final model in boys. Corresponding standardized coefficients were $0.211,0.218$, and 0.242 (all $p<0.05$ ), respectively (Table 2). However, the association between LPA and different categories of IQ were not found in girls in this study. Furthermore, the results were similar with those described above when LPA and MVPA were separately entered into the model; only LPA positively correlated with FIQ, solely in boys $(\beta=0.208$, $p<0.05)$. In addition, when TPA replaced LPA and MVPA into the model, the results also suggested TPA positively associated with VIQ, and FIQ, solely in boys ( $\beta=0.236$ and 0.179 , all $p<0.05)$.

Table 2. Linear regression analyses between cognitive function and physical activity.

\begin{tabular}{|c|c|c|c|c|c|c|}
\hline \multirow{2}{*}{ Predictor Variables } & \multicolumn{2}{|r|}{ VIQ } & \multicolumn{2}{|r|}{ PIQ } & \multicolumn{2}{|r|}{ FIQ } \\
\hline & $\beta$ & $95 \%$ CI & $\beta$ & $95 \%$ CI & $\beta$ & $95 \%$ CI \\
\hline \multicolumn{7}{|l|}{ Boys } \\
\hline \multicolumn{7}{|l|}{ Model $1 *$} \\
\hline LPA & 0.203 & $-0.009,0.407$ & 0.191 & $-0.031,0.416$ & 0.224 & $0.008,0.441$ \\
\hline MVPA & 0.162 & $-0.048,0.356$ & -0.001 & $-0.218,0.217$ & 0.082 & $-0.130,0.290$ \\
\hline$R^{2}$ & & 0.099 & & 0.022 & & 0.069 \\
\hline \multicolumn{7}{|l|}{ Model $2^{+}$} \\
\hline LPA & 0.197 & $0.009,0.377$ & 0.188 & $-0.019,0.398$ & 0.218 & $0.029,0.408$ \\
\hline MVPA & 0.064 & $-0.120,0.242$ & -0.076 & $-0.280,0.131$ & -0.016 & $-0.202,0.171$ \\
\hline$R^{2}$ & & 0.300 & & 0.152 & & 0.291 \\
\hline \multicolumn{7}{|l|}{ Model $3 \ddagger$} \\
\hline LPA & 0.211 & $0.018,0.395$ & 0.218 & $0.007,0.433$ & 0.242 & $0.048,0.435$ \\
\hline MVPA & 0.043 & $-0.150,0.232$ & -0.122 & $-0.335,0.096$ & -0.051 & $-0.245,0.146$ \\
\hline$R^{2}$ & & 0.297 & & 0.157 & & 0.293 \\
\hline \multicolumn{7}{|l|}{ Girls } \\
\hline \multicolumn{7}{|l|}{ Model 1* } \\
\hline LPA & -0.028 & $-0.271,0.212$ & 0.064 & $-0.161,0.289$ & 0.007 & $-0.225,0.240$ \\
\hline MVPA & -0.009 & $-0.265,0.245$ & 0.108 & $-0.124,0.353$ & 0.037 & $-0.206,0.286$ \\
\hline$R^{2}$ & & -0.017 & & 0.006 & & -0.016 \\
\hline \multicolumn{7}{|l|}{ Model $2^{+}$} \\
\hline LPA & -0.073 & $-0.311,0.156$ & 0.025 & $-0.205,0.255$ & -0.038 & $-0.268,0.191$ \\
\hline MVPA & -0.025 & $-0.276,0.220$ & 0.103 & $-0.134,0.353$ & 0.029 & $-0.212,0.274$ \\
\hline$R^{2}$ & & 0.090 & & 0.011 & & 0.060 \\
\hline \multicolumn{7}{|l|}{ Model $3 \ddagger$} \\
\hline LPA & -0.023 & $-0.252,0.203$ & 0.027 & $-0.206,0.261$ & -0.003 & $-0.232,0.226$ \\
\hline MVPA & -0.143 & $-0.414,0.092$ & 0.097 & $-0.157,0.363$ & -0.053 & $-0.312,0.196$ \\
\hline$R^{2}$ & & 0.157 & & 0.002 & & 0.087 \\
\hline
\end{tabular}

Note: $\beta$, standardized coefficients; $R^{2}$, adjusted R square; LPA, light physical activity; MVPA, moderate to vigorous physical activity; VIQ, Verbal Intelligence Quotient; PIQ, Performance Intelligence Quotient; FIQ, Full Intelligence Quotient; the $p$ values less than 0.05 are bolded; ${ }^{*}$ Model 1: unadjusted; ${ }^{\dagger}$ Model 2: adjusted for age, BMI status, mother's education, family structure, household income, and child behavior scores; $\ddagger$ Model 3: further adjusted for cardiorespiratory fitness, which was log-transformed before being entered into the model. 


\section{Discussion}

In this cross-sectional study, the major finding was that LPA was significantly and positively associated with intelligence quotient in boys, but not in girls. Based on the evidence available, the most recent systematic review concluded that there is a positive association between PA and cognitive function in children, although more studies are needed to identify the effects of different types, amounts, and frequencies of PA on cognitive function [6]. Moreover, in general, MVPA was recommended for children and adolescents because of the "intensity threshold" of PA benefit [21]. However, findings from this study are not in agreement with the results of previous studies, which found only LPA was evidently correlated with cognitive function, as measured by standardized IQ testing, in preschool children. A potential reason has been suggested to explain why different intensities of PA may play a different role among different age groups. One of the hypotheses of PA's effect on cognitive function was mediated by cardiorespiratory fitness [22], which increased responding to LPA in preschool children but may need higher intensity stimulation in children and adolescents. Nevertheless, it is not suggested that we can ignore the importance of MVPA, although only LPA is shown to be correlated with cognition in this study. Further, total minutes of PA (TPA, sum of LPA and MVPA) also presented a notable association with VIQ and FIQ when TPA took the place of LPA and MVPA in the model. Moreover, the current guidelines of PA recommend accumulating at least $180 \mathrm{~min}$ of PA daily at any intensity, and especially highlight the importance of TPA for preschool children [23]. Engaging in both LPA and MVPA lead to increases in the amount of TPA and, therefore, neither of them can be ignored.

The positive association between PA and cognitive functions solely found in boys in this study is also contrary to a recent review article, which showed the sex-dependent effect was more significant in girls [1]. A possible explanation for the findings was the lower level of cardiorespiratory fitness in girls at baseline which could result in more apparent physiological effect in the analysis [24]. Considering the possible influencing factors in this study, there were several explanations for our current results. First, boys engaged in more PA than girls, possibly having a dose-response effect, allowing boys to accrue greater cognitive function benefits. Especially, accumulating evidence from animal to human studies demonstrated that engaging in more physical activity can increase expression and concentration of brain-derived neurotrophic factor in hippocampus [25], which has been to play a crucial role in brain plasticity and functions [26]. Second, contrary to adolescents, preschool children, especially boys, were found to have lower levels of cardiorespiratory fitness in this study. This may have been a physiological effect derived from boys' PA stimulation. Furthermore, hypothalamic-pituitary-adrenal (HPA) axis response to PA is sex-dependent, possibly causing the sex-dependent effect [27]. For example, higher activation of HPA axis to PA in boys initiates a number of physiological changes, such as stimulating protein synthesis, which serves as the basis for a number of hormones, including adrenocorticotropic hormone (ACTH) and bendorphin that improve cognition, behavior response, and homeostatic challenges [28]. Additionally, sex difference is also a result of genetic variation, parental and familial factors, and one's acquired behaviors and perceptions, with the latter often shaped by unique experiences at the individual, parental, and familial levels [29].

Our study has several strengths. First, PA was measured using an accelerometer, which avoided the recall bias of proxy report by parents or teachers. Second, several potential confounding factors were adjusted in the statistical analyses. Third, our results were strengthened by combining and separating LPA and MVPA into linear regression models. However, our study also has several limitations. First, for feasibility, we used a convenience sample in this observational study. Second, our cross-sectional study design has limited ability to draw a causal relation of our findings. We cannot illustrate whether PA improves cognitive functions in boys or whether preschool aged boys with high levels of cognitive function simply tend to participant in more PA. Last, the accelerometer was worn over the hip, which limited the ability to capture activities with little displacement of the body, such as cycling. However, the hip was probably the best placement to capture whole-body movements and was also the site most often used by various studies [30]. 


\section{Conclusions}

In conclusion, our findings suggest PA has a significant and positive association with cognitive functions in boys, especially LPA. On the basis of this study, we indicated the benefit of cognitive function derived from LPA and recommend sex-dependent responses should be considered in future studies. Moreover, more prospective and intervention studies are needed to clarify the causal relation and mechanisms of our observed sex-specific effect.

Author Contributions: M.Q. conceived and designed the study, analyzed the data and drafted the manuscript. H.Z., J.Z., T.Z., J.Z., G.Z., H.F. and S.S. conducted the experiments and collected the data. M.Q. and G.Z. performed the literature search. R.W. and P.C. advised on analysis and interpretation of the data, and critically revised the manuscript.

Acknowledgments: This work was supported by the Science and Technology Commission of Shanghai Municipality, China (12XD1404500); the National Natural Science Foundation of China (81703252); the Humanity and Social Science Youth foundation of Ministry of Education of China (15YJC890029); and a grant from the Shanghai Municipal Education Committee (HJTY-2014-A10).

Conflicts of Interest: The authors declare no conflicts of interest.

\section{References}

1. Esteban-Cornejo, I.; Tejero-Gonzalez, C.M.; Sallis, J.F.; Veiga, O.L. Physical activity and cognition in adolescents: A systematic review. J. Sci. Med. Sport 2015, 18, 534-539. [CrossRef] [PubMed]

2. Moffitt, T.E.; Arseneault, L.; Belsky, D.; Dickson, N.; Hancox, R.J.; Harrington, H.L.; Houts, R.; Poulton, R.; Roberts, B.W.; Ross, S. A gradient of childhood self-control predicts health, wealth, and public safety. Proc. Natl. Acad. Sci. USA 2011, 108, 2693-2698. [CrossRef] [PubMed]

3. Carvalho, A.; Rea, I.M.; Parimon, T.; Cusack, B.J. Physical activity and cognitive function in individuals over 60 years of age: A systematic review. Clin. Interv. Aging 2014, 9, 661-682. [PubMed]

4. Moreau, D.; Kirk, I.J.; Waldie, K.E. High-intensity training enhances executive function in children in a randomized, placebo-controlled trial. eLife 2017, 6, e25062. [CrossRef] [PubMed]

5. Benzing, V.; Heinks, T.; Eggenberger, N.; Schmidt, M. Acute cognitively engaging exergame-based physical activity enhances executive functions in adolescents. PLoS ONE 2016, 11, e0167501. [CrossRef] [PubMed]

6. Donnelly, J.E.; Hillman, C.H.; Castelli, D.; Etnier, J.L.; Lee, S.; Tomporowski, P.; Lambourne, K.; Szabo-Reed, A.N. This summary was written for the American College of Sports Medicine, b. Physical activity, fitness, cognitive function, and academic achievement in children: A systematic review. Med. Sci. Sports Exerc. 2016, 48, 1223-1224. [CrossRef] [PubMed]

7. Dishman, R.K.; Berthoud, H.R.; Booth, F.W.; Cotman, C.W.; Edgerton, V.R.; Fleshner, M.R.; Gandevia, S.C.; Gomez-Pinilla, F.; Greenwood, B.N.; Hillman, C.H. Neurobiology of exercise. Obesity 2006, 14, 345-356. [CrossRef] [PubMed]

8. Davis, C.L.; Tomporowski, P.D.; Mcdowell, J.E.; Austin, B.P.; Miller, P.H.; Yanasak, N.E.; Allison, J.D.; Naglieri, J.A. Exercise improves executive function and achievement and alters brain activation in overweight children: A randomized controlled trial. Health Psychol. Off. J. Div. Health Psychol. Am. Psychol. Assoc. 2011, 30, 91-98. [CrossRef] [PubMed]

9. Carson, V.; Hunter, S.; Kuzik, N.; Wiebe, S.A.; Spence, J.C.; Friedman, A.; Tremblay, M.S.; Slater, L.; Hinkley, T. Systematic review of physical activity and cognitive development in early childhood. J. Sci. Med. Sport 2016, 19, 573-578. [CrossRef] [PubMed]

10. Liu, J.; Zhou, G.; Wang, Y.; Ai, Y.; Pinto-Martin, J.; Liu, X. Sleep problems, fatigue, and cognitive performance in chinese kindergarten children. J. Pediatr. 2012, 161, 520-525.e2. [CrossRef] [PubMed]

11. Santos, D.N.; Assis, A.M.; Bastos, A.C.; Santos, L.M.; Santos, C.A.; Strina, A.; Prado, M.S.; Almeida-Filho, N.M.; Rodrigues, L.C.; Barreto, M.L. Determinants of cognitive function in childhood: A cohort study in a middle income context. BMC Public Health 2008, 8, 202. [CrossRef] [PubMed]

12. Zhao, G.; Quan, M.; Su, L.; Zhang, H.; Zhang, J.; Zhang, J.; Fang, H.; Cao, Z.; Zhu, Z.; Niu, Z. Effect of physical activity on cognitive development: Protocol for a 15-year longitudinal follow-up study. Biomed. Res. Int. 2017, 2017, 8568459. [CrossRef] [PubMed] 
13. Gong, Y.-X.; Dai, X.-Y. China-wechsler younger children scale of intelligence (c-wycsi). Psychol. Sci. 1986, 2, 23-30.

14. Kramer, M.S.; Aboud, F.; Mironova, E.; Vanilovich, I.; Platt, R.W.; Matush, L.; Igumnov, S.; Fombonne, E.; Bogdanovich, N.; Ducruet, T.; et al. Breastfeeding and child cognitive development: New evidence from a large randomized trial. Arch. Gen. Psychiatry 2008, 65, 578-584. [CrossRef] [PubMed]

15. Li, X.; Atkins, M.S. Early childhood computer experience and cognitive and motor development. Pediatrics 2004, 113, 1715-1722. [CrossRef] [PubMed]

16. Pate, R.R.; Almeida, M.J.; McIver, K.L.; Pfeiffer, K.A.; Dowda, M. Validation and calibration of an accelerometer in preschool children. Obesity 2006, 14, 2000-2006. [CrossRef] [PubMed]

17. Cain, K.L.; Sallis, J.F.; Conway, T.L.; Van Dyck, D.; Calhoon, L. Using accelerometers in youth physical activity studies: A review of methods. J. Phys. Act. Health 2013, 10, 437-450. [CrossRef] [PubMed]

18. Cole, T.J.; Bellizzi, M.C.; Flegal, K.M.; Dietz, W.H. Establishing a standard definition for child overweight and obesity worldwide: International survey. BMJ 2000, 320, 1240-1243. [CrossRef] [PubMed]

19. Ek, A.; Sorjonen, K.; Nyman, J.; Marcus, C.; Nowicka, P. Child behaviors associated with childhood obesity and parents' self-efficacy to handle them: Confirmatory factor analysis of the lifestyle behavior checklist. Int. J. Behav. Nutr. Phys. Act. 2015, 12, 36. [CrossRef] [PubMed]

20. Ortega, F.B.; Cadenas-Sánchez, C.; Sánchez-Delgado, G.; Mora-González, J.; Martínez-Téllez, B.; Artero, E.G.; Castro-Piñero, J.; Labayen, I.; Chillón, P.; Löf, M. Systematic review and proposal of a field-based physical fitness-test battery in preschool children: The prefit battery. Sports Med. 2015, 45, 533-555. [CrossRef] [PubMed]

21. Kwak, L.; Kremers, S.P.; Bergman, P.; Ruiz, J.R.; Rizzo, N.S.; Sjöström, M. Associations between physical activity, fitness, and academic achievement. J. Pediatr. 2009, 155, 914-918.e1. [CrossRef] [PubMed]

22. Suwabe, K.; Hyodo, K.; Byun, K.; Ochi, G.; Fukuie, T.; Shimizu, T.; Kato, M.; Yassa, M.A.; Soya, H. Aerobic fitness associates with mnemonic discrimination as a mediator of physical activity effects: Evidence for memory flexibility in young adults. Sci. Rep. 2017, 7, 5140. [CrossRef] [PubMed]

23. Tremblay, M.S.; Chaput, J.P.; Adamo, K.B.; Aubert, S.; Barnes, J.D.; Choquette, L.; Duggan, M.; Faulkner, G.; Goldfield, G.S.; Gray, C.E. Canadian 24-h movement guidelines for the early years (0-4 years): An integration of physical activity, sedentary behaviour, and sleep. BMC Public Health 2017, 17, 874. [CrossRef] [PubMed]

24. Carlson, S.A.; Fulton, J.E.; Lee, S.M.; Maynard, L.M.; Brown, D.R.; Kohl, H.W., 3rd.; Dietz, W.H. Physical education and academic achievement in elementary school: Data from the early childhood longitudinal study. Am. J. Public Health 2008, 98, 721-727. [CrossRef] [PubMed]

25. Zoladz, J.A.; Pilc, A. The effect of physical activity on the brain derived neurotrophic factor: From animal to human studies. J. Physiol. Pharmacol. 2010, 61, 533-541. [PubMed]

26. Park, H.; Poo, M.M. Neurotrophin regulation of neural circuit development and function. Nat. Rev. Neurosci. 2013, 14, 7-23. [CrossRef] [PubMed]

27. Baker, L.D.; Frank, L.L.; Foster-Schubert, K.; Green, P.S.; Wilkinson, C.W.; McTiernan, A.; Plymate, S.R.; Fishel, M.A.; Watson, G.S.; Cholerton, B.A. Effects of aerobic exercise on mild cognitive impairment: A controlled trial. Arch. Neurol. 2010, 67, 71-79. [CrossRef] [PubMed]

28. Lee, R.S.; Sawa, A. Environmental stressors and epigenetic control of the hypothalamic-pituitary-adrenal axis. Neuroendocrinology 2014, 100, 278-287. [CrossRef] [PubMed]

29. Ogilvie, K.M.; Rivier, C. Gender difference in hypothalamic-pituitary-adrenal axis response to alcohol in the rat: Activational role of gonadal steroids. Brain Res. 1997, 766, 19-28. [CrossRef]

30. Cliff, D.P.; Reilly, J.J.; Okely, A.D. Methodological considerations in using accelerometers to assess habitual physical activity in children aged 0-5 years. J. Sci. Med. Sport 2009, 12, 557-567. [CrossRef] [PubMed]

(C) 2018 by the authors. Licensee MDPI, Basel, Switzerland. This article is an open access article distributed under the terms and conditions of the Creative Commons Attribution (CC BY) license (http:/ / creativecommons.org/licenses/by/4.0/). 